\title{
Forecast Global Carbon Dioxide Emission Using Swarm Intelligence
}

\author{
Alaa Abdelfatah, Sahar A. Mokhtar, Alaa Sheta \\ Computers and Systems Department \\ Electronics Research Institute (ERI) \\ Cairo, Egypt
}

\author{
Basma Solaiman \\ College of Computer Science and Information Technology \\ Sudan University of Science and Technology (SUST) \\ Khartoum, Sudan
}

\begin{abstract}
The tremendous effects of air quality in large cities have been considered a severe environmental problem all over the world. Therefore, the international community agreed to develop air quality standards to monitor and control pollution rates around industrial communities. Harmful emission into the air is a sign that could extremely affect man's health, natural life and agriculture. Forecasting models is essential for predicting air quality. $\mathrm{CO}_{2}$ emissions have been an international concern because of fossil fuels. In this study, Particle Swarm Optimization (PSO) is used for analyzing world $\mathrm{CO}_{2}$ emission based on the global energy consumption. A parametric PSO model is developed to forecast $\mathrm{CO}_{2}$ emission based set of attributes. They include: global oil, natural gas, coal, and primary energy consumption. A data set collected during the years 1980 and 2010 were used in this study. Experimental results show that PSO can provide good modeling results using a limited number of measurements compared to other linear models.
\end{abstract}

\section{General Terms:}

Climate Change, Carbon Dioxide Emission, Particle Swarm Optimization

\section{Keywords:}

Air Pollution, Carbon Emission, Swarm Intelligence

\section{INTRODUCTION}

Climate change towards the increase of air pollution presented a significant environmental harmfulness. This reason causes an increased interest from both academics and policy-makers in developing prediction model in the area of $\mathrm{CO} 2$ emissions. Air quality prediction model helps in the management of our environment. The air pollution prediction is a complex nonlinear process function of numerous parameters [16, 13, 17]. Air quality information is collected from various sources and sensors to be used in developing a successful relationship which helps producing accurate prediction models. Mathematical methods and tools were introduced to solve variety of problems in system identification, control, stock market prediction and air pollution modeling [10]. The complexity of air pollution data has been broadly discussed in [15], while the usage of various modeling tools was addressed in related literature [13], [21]. In [3], authors used a new panel data set covering the fifty United States and Washington D.C. from 1960-2001 to model $\mathrm{CO}_{2}$ emission. Their objective was to measure of model performance using the squared out-of-sample prediction error of aggregate $\mathrm{CO}_{2}$ emissions. $\mathrm{CO}_{2}$ emissions and economic growth in Nigeria for the period 1971-2009, in a multivariate framework was presented in [8]. Authors in [19] provided a study to develop statistical models for predicting the carbon dioxide emissions and the atmosphere in the United States. A monthly emissions data from 1981 to 2003 that was collected by the Carbon Dioxide Information Analysis Center was used. The developed statistical models took into consideration various trends and seasonal effects. Forecasting the expected growth of China's $\mathrm{CO}_{2}$ emissions using province-level information was presented in [2].

Air pollution has been explored in many articles based soft computing and computational intelligence techniques [15, 4]. For example, in [1] authors develop a non-parametric Artificial Neural Network (ANN) models to predict both the Particulate Matters (PM10) and Total Suspended Particles (TSP) in Salt, Jordan. Two Artificial Neural Network based AutoRegressive with eXternal (ANNARX) Input models were used to provide high performance modeling for the PM10 and the TSP air pollution parameters. An integrated multi-layer perceptron neural network and Bees Algorithm was proposed to analyze the world $\mathrm{CO}_{2}$ emissions [5]. A data set from the years 1980 to 2006 were used, the data for the years 1980-1999 were used for training and the testing data used was for the years 2000-2006. The model was used to predict the World CO2 emission up to year 2040. Several models have been proposed to investigate the causal relationships between energy consumption and $\mathrm{CO}_{2}$ emission. For instance, the relatively new time series technique known as the Toda-Yamamoto method [12], and the Grey prediction model (GM) [17], have been applied to predict $\mathrm{CO}_{2}$ levels. Neural networks have been used for short-term $\mathrm{CO}_{2}$ prediction [5]. In addition methods like Genetic Algorithm (GA) [11] have been employed for predicting $\mathrm{CO}_{2}$ emission concentrations.

In this paper, our objective is to develop a mathematical model for the global $\mathrm{CO}_{2}$ emission based on a set of variables. They include global oil, natural gas, coal and primary energy consumption over the period from 1980 to 2010 using a Particle Swarm Optimization (PSO). The proposed model should be able to provide an accurate estimation results in our case. 


\section{AIR POLLUTION}

Fossil energy resources remain abundant but contain significant amounts of carbon that are normally released during combustion. The proven and probable reserves of oil and gas are enough to last for decades and in the case of coal for centuries. Possible undiscovered resources extend these projections even further. Fossil energy use is responsible for about $85 \%$ of the anthropogenic $\mathrm{CO}_{2}$ emissions produced annually [14]. In Figure 1, we show the origin of $\mathrm{CO}_{2}$ emissions from all fossil fuel combustion by region of the world. It was found that almost half of the total emissions come from Organization of Economic Cooperation and Development (OECD) countries, excluding Mexico, and only 20 percent are emitted in China, but only seven percent are from Latin America.

\section{PARTICLE SWARM OPTIMIZATION}

Particle Swarm Optimization (PSO) was developed in 1995 by James Kennedy (social-psychologist), and Russell Eberhart (electrical engineer) [9]. PSO is a robust stochastic nonlinearoptimization technique based on movement and intelligence of swarms. It is inspired from social behavior of bird or fish, where a group of birds randomly search for food in an area by following the nearest bird to the food. It combines local search methods with global search methods (exploration and exploitation). Moreover, it depends on social interaction between swarms to locate the best achieved position so far. The main idea of PSO is obtaining a number of particles that constitute a swarm moving around in the search space looking for the best solution. Each particle is treated as a point in a multi-dimensional space. The particles move around in the search space, and communicate either directly or indirectly with one another.

In the implementation of PSO, each particle tries to modify its position using information as: its current position, its current velocity, the distance between the current position and best solution individually found, and the distance between the current position and the best solution found in its neighborhood [7]. The basic PSO equations are given as follows:

$$
v_{i d}^{\text {new }}=v_{i d}^{\text {old }}+\phi_{1} * c_{1} *\left(p_{i d}-x_{i d}\right)+\phi_{2} * c_{2} *\left(p_{g d}-x_{i d}\right)
$$

$$
x_{i d}^{n e w}=x_{i d}^{\text {old }}+v_{i d}^{\text {new }}
$$

where:

$v_{i d}$ represents the velocity of particle $i$ in dimension $d$,

$x_{i d}$ represents the position of particle $i$ in dimension $d$,

$\phi_{1}, \phi_{2}$ are positive constants,

$c_{1}, c_{2}$ are random numbers

$p_{i d}$ is the best position reached so far by the particle, and

$p_{g d}$ is the global best position reached by the neighborhood.

The previously shown velocity update equation is influenced by two components: $\left(p_{i d}-x_{i d}\right)$ that represents the cognitive component, and $\left(p_{g d}-x_{i d}\right)$ that represent the social component. The velocity of PSO is prune to reach infinity which lead to causing the particles to reach a state of instability. Therefore, the velocity of the particle should not exceed a maximum value, $v_{\max }$. The performance can suffer if maximum velocity is inappropriately set. If it is too high, the particles can fly past optimal solutions, and if it is too low, they can get stuck in local minimal. More enhancements on the basic PSO model are stated in [20].
The PSO algorithm is straightforward (see Algorithm 11. First, initialize particles with random position and velocity vectors. For each particle: evaluate the fitness and if it is better than the best individual fitness then update it. After that, update the best global fitness. Then obtain the new velocity and position for each particle. This procedure is repeated for a number of iterations (epochs) or until convergence is beyond a certain limit.

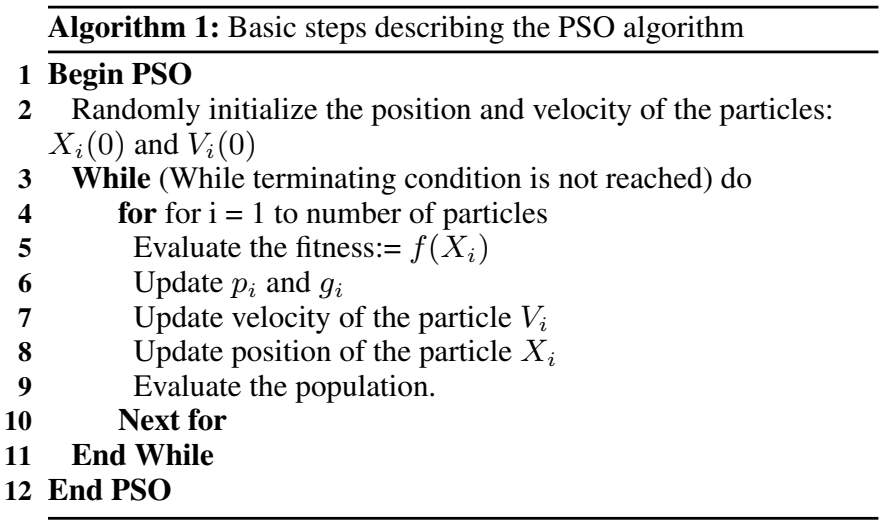

\subsection{Nature of Data}

The Particle Swarm Optimization (PSO) model was used to forecast the $\mathrm{CO}_{2}$ emission using the four energy commodities that are considered the highest. Long-term (annual) forecasting was performed. The related data from 1980 to 2010 were used, partly for installing the models (finding candidates of best weighting factors for each model (1980-2003) and partly for testing the models (2004 to 2010). The values of global oil, natural gas, coal, and primary energy consumption are presented in [11.18].

\subsection{Evaluation Criterion}

In order to check the performance of the developed PSO model, Route Mean Square (RMS), the Euclidian distance (ED), the Manhattan distance (MD) and the Variance-Accounted-For (VAF) were measured. These performance criteria were assessed to measure how close the measured values to those developed using the PSO approach. This criterion helps in better evaluating the capabilities of the developed PSO model. The RMS, VAF, ED and MD are computed as follows:

(1) Route Mean Square (RMS):

$$
R M S=\sqrt{\frac{\sum_{i=1}^{n}\left|\left(y_{i}-\hat{y}_{i}\right)\right|}{n}}
$$

(2) Variance-Accounted-For (VAF)

$$
\left.V A F=\left[1-\frac{\operatorname{var}(y-\hat{y})}{\operatorname{var}(y)}\right)\right] \times 100 \%
$$

(3) Euclidian distance (ED):

$$
E D=\sqrt{\sum_{i=1}^{n}\left(y_{i}-\hat{y}_{i}\right)^{2}}
$$




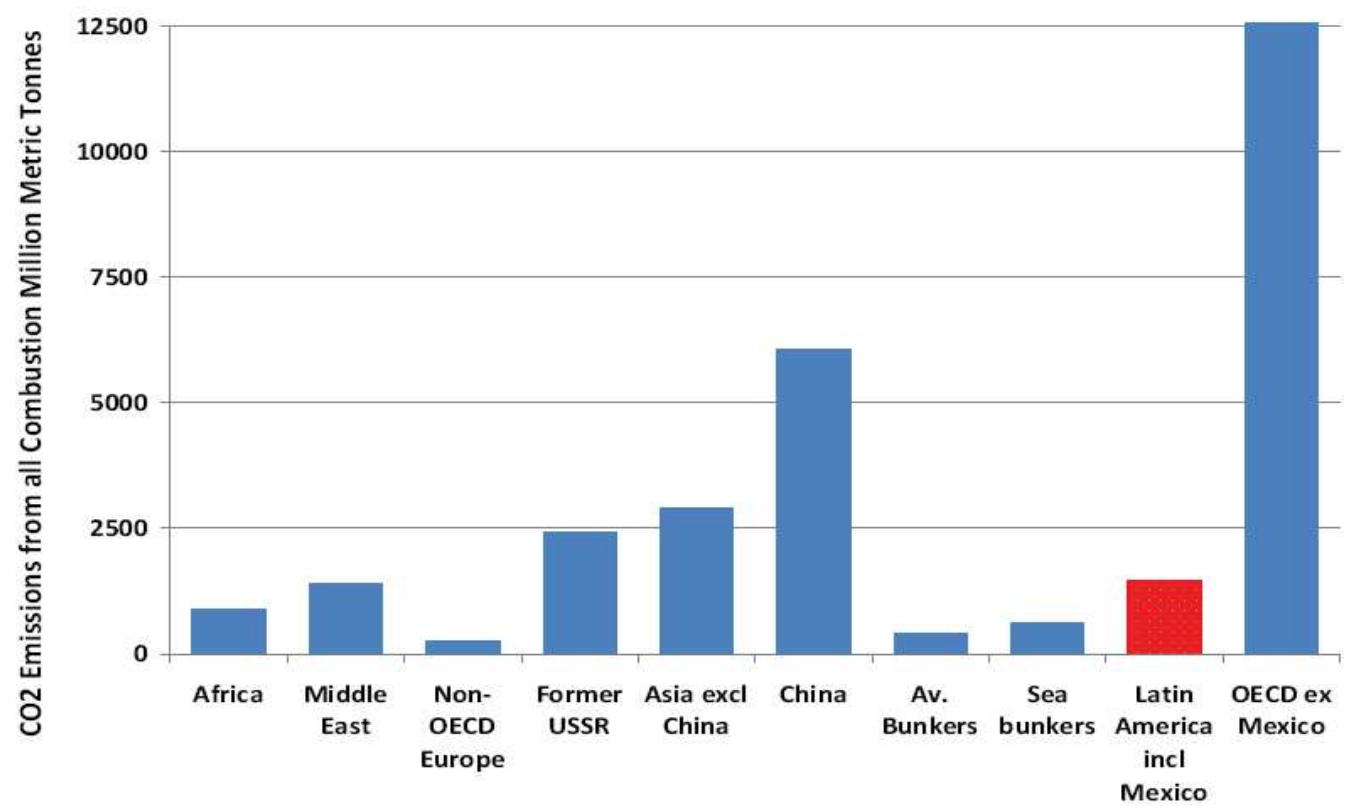

Fig. 1. $\mathrm{CO}_{2}$ emissions from all fossil fuel combustion by country or region in 2006 (million metric tonnes).Source: International Energy Agency (IEA2008)

(4) Manhattan distance (MD):

$$
M D=\left(\sum_{i=1}^{n}\left|y_{i}-\hat{y}_{i}\right|\right)
$$

where $y$ and $\hat{y}$ are the observed and estimated $\mathrm{CO}_{2}$ of the proposed model and $n$ is the number of measurements used in the experiments, respectively.

\section{EXPERIMENTAL RESULTS}

The input and output data used for the model is presented in Table 1. The proposed model is an exponential model of the input variables. The tuning parameters and search space for PSO is given in Table 2 A Matlab Toolbox for PSO was used to develop our results [6]. Candidate solutions (particles) in this case are just ndimensional vectors of particles of the form:

$$
\begin{array}{|l|l|l|l|l|l|l|l|l|}
\hline \alpha_{1} & \beta_{1} & \alpha_{2} & \beta_{2} & \alpha_{3} & \beta_{3} & \alpha_{4} & \beta_{4} & \gamma \\
\hline
\end{array}
$$

The search process begins by random distribution of the initial PSO population which represents a random sample of the PSO search space. The corresponding fitness of each particle is computed. Particles with higher fitness shall be selected to produce new population which enhances the features of their parents. The problem under consideration is to estimate the correct parameters $\alpha_{1}, \beta_{1}, \alpha_{2}, \beta_{2}, \alpha_{3}, \beta_{3}, \alpha_{4}$ and $\beta_{4}$ for the proposed exponential model.

A data set adopted from [11] for the model development process was used. We defined the parameter space and other PSO setup tuning parameters are given in Table 2 . The fitness (i.e. quality) of a particular estimate is obtained by observing the behavior of the system with the estimated parameters, and using the RMS error between the actual and predicted $\mathrm{CO}_{2}$.

$$
C O_{2}=\alpha_{1} O i l^{\beta_{1}}+\alpha_{2} N G^{\beta_{2}}+\alpha_{3} \text { Coal }^{\beta_{3}}+\alpha_{4} P E^{\beta_{4}}+\gamma(7
$$

Table 1. Inputs and Output for the Exponential PSO model

\begin{tabular}{|l|llll|}
\hline Inputs & $\mathrm{Oil}, \quad \mathrm{NG}, \quad$ Coal, & $\mathrm{PE}$ \\
Output & $\mathrm{CO}_{2}$ & & & \\
\hline
\end{tabular}

Table 2. The tuning parameters for the PSO

\begin{tabular}{|ll|}
\hline Operator & Value \\
\hline \hline Acceleration constant & {$[2.1,2.1]$} \\
Inertia Weight & {$[0.9,0.6]$} \\
Maximum no. of Iteration & 1000 \\
Maximum Velocity & 150 \\
No. of Particles & 9 \\
Search space for $\alpha$ and $\beta$ & {$[-10,10]$} \\
Search space for $\gamma$ & {$[-1000,1000]$} \\
\hline
\end{tabular}

The computed model parameters using PSO was found as given in Equation 8

$$
\begin{aligned}
\mathrm{CO}_{2} & =8.0639 \mathrm{Oil}^{-4.2278}+5.9998 \mathrm{NG}^{-0.63625} \\
& +4.4493 \mathrm{Coal}^{-0.14562}+2.4769 \mathrm{PE}^{1.1308}+3.7837
\end{aligned}
$$

Previous research work presented in [11] explored the use of Genetic Algorithms to find the best tuning parameters for the same exponential model. The developed model is presented in Equation 9

$$
\begin{aligned}
\mathrm{CO}_{2} & =0.3427 \mathrm{Oil}^{2.0509}+0.9188 N G^{0.9115} \\
& +0.0092 \text { Coal }^{0.9096}+0.25644 \mathrm{PE}^{0.2827}+0.3597
\end{aligned}
$$



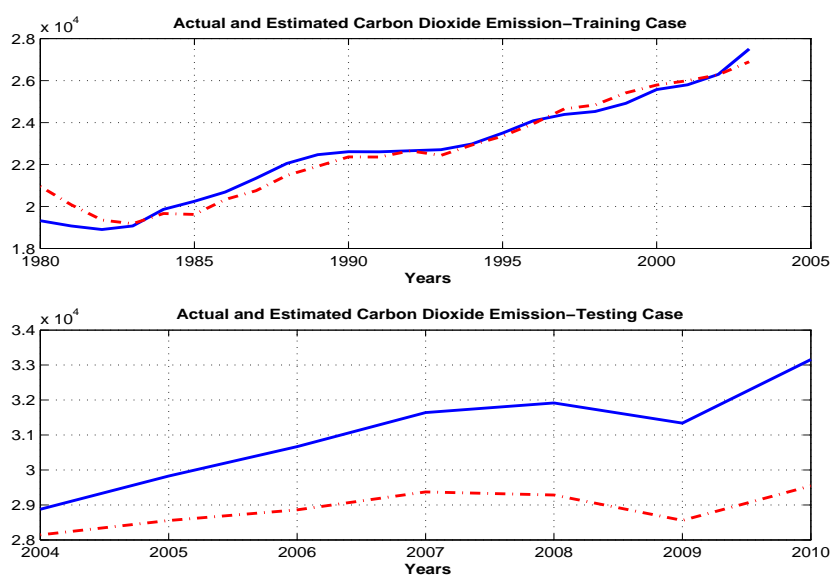

Fig. 2. Observed and Estimated Exponential PSO Model

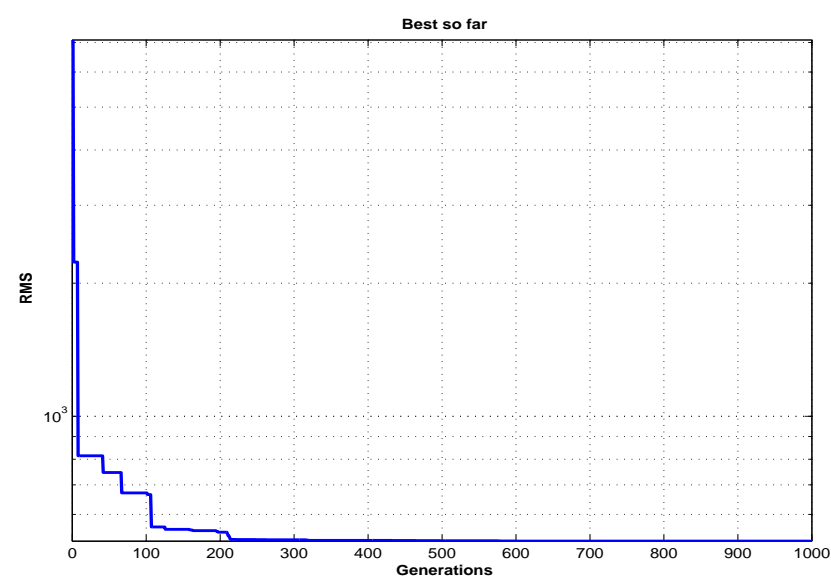

Fig. 3. RMS convergence of the PSO process

Table 3. Evaluation criteria of the PSO model

\begin{tabular}{|c|l|l|l|l|}
\hline Criteria & RMS & VAF & ED & MD \\
\hline \hline Training & 537.86 & $92.978 \%$ & 2464.8 & 401.14 \\
\hline Testing & 2121.4 & $72.913 \%$ & 6708.4 & 1740.2 \\
\hline
\end{tabular}

It was found that the model developed using PSO is much stable and can provide better evaluation criterion which was adopted in this study.

\section{CONCLUSIONS}

This paper provided an exponential model for the global carbon emission based on global oil, natural gas, coal, and primary energy consumption attributes. A data set of the years (19802010) was used for estimating nine parameters of the model using PSO in both the training and testing cases. The developed results showed the advantages of PSO in tuning the model than GAs. Future work will focus on exploring the advantage of evolutionary computation techniques such as genetic programming to develop a mathematical model for the global carbon emission.

\section{ACKNOWLEDGEMENT}

Basma Solaiman is a PhD student with the College of Computer Science and Information Technology, Sudan University of Science and Technology (SUST), Khartoum, Sudan. She is on leave from the New and Renewable Energy Authority (NREA), Cairo, Egypt.

\section{REFERENCES}

[1] Mouhammd Al-kasassbeh, Alaa F. Sheta, Hossam Faris, and Hamza Turabieh. Prediction of pm10 and tsp air pollution parameters using artificial neural network autoregressive, external input models: A case study in salt, jordan. MiddleEast Journal of Scientific Research, 14(7):999-1009, 2013.

[2] Maximilian Auffhammer and Richard T. Carson. Forecasting the path of china's $\mathrm{CO} 2$ emissions using province-level information. Journal of Environmental Economics and Management, 55(3):229-247, May 2008.

[3] Maximilian Auffhammer and Ralf Steinhauser. Forecasting the path of U.S. CO2 emissions using state-level information. The Review of Economics and Statistics, 94(1):172-185, February 2012.

[4] A. Baareh. Solving the carbon dioxide emission estimation problem: An artificial neural network model. Journal of Software Engineering and Applications, 6(7):338-342, 2013.

[5] M. A. Behrang, E. Assareh, M. R. Assari, and A. Ghanbarzadeh. Using bees algorithm and artificial neural network to forecast world carbon dioxide emission. Energy Sources, Part A: Recovery, Utilization, and Environmental Effects, 33(19):1747-1759, 2011.

[6] B. Birge. PSOt - a particle swarm optimization toolbox for use with Matlab. In Proceedings of the 2003 IEEE Swarm Intelligence Symposium (SIS03), pages 182-186, 2003.

[7] D. Bratton and J. Kennedy. Defining a standard for particle swarm optimization. In IEEE Swarm Intelligence Symposium, pages $120-127,2007$.

[8] E. Nnaji Chibueze, O. Chukwu Jude, and Nnaji Moses. Electricity supply, fossil fuel consumption, $\mathrm{CO} 2$ emissions and economic growth: Implications and policy options for sustainable development in nigeria. International Journal of Energy Economics and Policy, 3(3):262-271, 2013.

[9] Andries P. Engelbrecht. Computational Intelligence: An Introduction. Wiley Publishing, 2nd edition edition, 2007.

[10] Kostas D. Karatzas, George Papadourakis, and Ioannis Kyriakidis. Understanding and forecasting atmospheric quality parameters with the aid of ANNs. In IEEE International Joint Conference on Neural Networks (IJCNN 2008), pages 2580-2587. IEEE, 2008.

[11] H Kavoosi, MH Saidi, M Kavoosi, and M Bohrng. Forecast global carbon dioxide emission by use of genetic algorithm. International Journal of Computer Science Issues (IJCSI), 9(1), 2012.

[12] Mohammad Reza Lotfalipour, Mohammad Ali Falahi, and NULL NULL. Economic growth, $\mathrm{CO} 2$ emissions, and fossil fuels consumption in Iran. Energy, (35):5115-5120, October 2010.

[13] Marek Makowski. Modeling paradigms applied to the analysis of european air quality. European Journal of Operational Research, 122(2):219-241, 2000. 
Table 4. Actual and Estimated $\mathrm{CO}_{2}$ Values Using the Exponential PSO Model

\begin{tabular}{|c|c|c|c|c|c|c|}
\hline Year & $\begin{array}{c}\text { Oil } \\
\text { Consumption } \\
(\text { Mtoe })^{a}\end{array}$ & $\begin{array}{c}\mathrm{NG} \\
\text { Consumption } \\
(\text { Mtoe })\end{array}$ & $\begin{array}{c}\text { Coal } \\
\text { Consumption } \\
(M \text { toe })\end{array}$ & $\begin{array}{c}\mathrm{PE} \\
\text { Consumption } \\
(\text { Mtoe })\end{array}$ & $\begin{array}{c}\mathrm{CO}_{2} \\
\text { Emission } \\
(M t)^{b}\end{array}$ & $\begin{array}{c}\mathrm{CO}_{2} \\
\text { Estimated } \\
(\mathrm{Mt})^{b}\end{array}$ \\
\hline 1980 & 2972.2 & 1296.9 & 1806.4 & 6624 & 19322.4 & 20953 \\
\hline 1981 & 2863 & 1309.5 & 1820.6 & 6577.5 & 19073.2 & 20084 \\
\hline 1982 & 2770.7 & 1312.5 & 1846.9 & 6548.4 & 18900.7 & 19354 \\
\hline 1983 & 2748.3 & 1329 & 1897.7 & 6638.2 & 19072.1 & 19177 \\
\hline 1984 & 2810.1 & 1440 & 1983.2 & 6960.2 & 19861 & 19665 \\
\hline 1985 & 2804.7 & 1488.3 & 2056 & 7137.5 & 20246.7 & 19623 \\
\hline 1986 & 2894.1 & 1503.6 & 2089.2 & 7307.5 & 20688.3 & 20331 \\
\hline 1987 & 2946.8 & 1579.6 & 2169 & 7555.7 & 21344.5 & 20750 \\
\hline 1988 & 3038.8 & 1654.9 & 2231.7 & 7833.5 & 22052.2 & 21484 \\
\hline 1989 & 3093 & 1729.2 & 2251.2 & 8001.7 & 22470.2 & 21918 \\
\hline 1990 & 3148.6 & 1769.5 & 2220.3 & 8108.7 & 22613.2 & 22364 \\
\hline 1991 & 3148.2 & 1807.5 & 2196.4 & 8156 & 22606.5 & 22360 \\
\hline 1992 & 3184.8 & 1817.9 & 2174.6 & 8187.6 & 22656.7 & 22655 \\
\hline 1993 & 3158 & 1853.9 & 2187.6 & 8257.5 & 22710.6 & 22439 \\
\hline 1994 & 3218.7 & 1865.4 & 2201.9 & 8357.6 & 22980.3 & 22927 \\
\hline 1995 & 3271.3 & 1927 & 2256.2 & 8577.9 & 23501.7 & 23351 \\
\hline 1996 & 3344.9 & 2020.5 & 2292.2 & 8809.5 & 24089.8 & 23946 \\
\hline 1997 & 3432.2 & 2016.8 & 2301.8 & 8911.6 & 24387.1 & 24654 \\
\hline 1998 & 3455.4 & 2050.3 & 2300.2 & 8986.6 & 24530.5 & 24842 \\
\hline 1999 & 3526 & 2098.4 & 2316 & 9151.4 & 24922.7 & 25417 \\
\hline 2000 & 3571.6 & 2176.2 & 2399.7 & 9382.4 & 25576.9 & 25789 \\
\hline 2001 & 3597.2 & 2216.6 & 2412.4 & 9465.6 & 25800.8 & 25998 \\
\hline 2002 & 3632.3 & 2275.6 & 2476.7 & 9651.8 & 26301.3 & 26285 \\
\hline 2003 & 3707.4 & 2353.1 & 2677.3 & 9997.8 & 27508.7 & 26900 \\
\hline 2004 & 3858.7 & 2431.8 & 2858.4 & 10482 & 28875.2 & 28145 \\
\hline 2005 & 3908.5 & 2511.2 & 3012.9 & 10800.9 & 29826.1 & 28556 \\
\hline 2006 & 3945.3 & 2565.6 & 3164.5 & 11087.8 & 30667.6 & 28860 \\
\hline 2007 & 4007.3 & 2661.3 & 3305.6 & 11398.4 & 31641.2 & 29373 \\
\hline 2008 & 3996.5 & 2731.4 & 3341.7 & 11535.8 & 31915.9 & 29284 \\
\hline 2009 & 3908.7 & 2661.4 & 3305.6 & 11363.2 & 31338.8 & 28557 \\
\hline 2010 & 4028.1 & 2858.1 & 3555.8 & 12002.4 & 33158.4 & 29545 \\
\hline
\end{tabular}

[14] Bert Metz. Climate Change 2007-Mitigation of Climate Change: Working Group III Contribution to the Fourth Assessment Report of the IPCC, volume 4. Cambridge University Press, 2007.

[15] Francesco Carlo Morabito and Mario Versaci. Fuzzy neural identification and forecasting techniques to process experimental urban air pollution data. Neural Networks, 16(34):493-506, 2003.

[16] A. Newcomer, S. Blumsack, J. Apt, L.B. Lave, and M.G. Morgan, editors. Electricity Load and Carbon Dioxide Emissions: Effects of a Carbon Price in the Short Term. 41st Hawaii International Conference on System Sciences, Computer Society Press, 2008.

[17] Hsiao-Tien Pao and Chung-Ming Tsai. Modeling and forecasting the $\mathrm{CO} 2$ emissions, energy consumption, and economic growth in brazil. Energy, 36(5):2450 - 2458, 2011.

[18] Reza Samsami. Application of ant colony optimization (ACO) to forecast $\mathrm{CO} 2$ emission in Iran. International Journal of Environment, Pharmacology and Life Sciences, Bulletin of Environment, Pharmacology and Life Sciences, 2:95-99, 2013.

[19] Shou Hsing Shih and Chris P. Tsokos. Prediction models for carbon dioxide emissions and the atmosphere. Neural, Parallel Sci. Comput., 16(1):165-178, January 2008.
[20] Ioan Cristian Trelea. The particle swarm optimization algorithm: convergence analysis and parameter selection. Information Processing Letters, 85(6):317-325, 2003.

[21] Takashi Washio, Einoshin Suzuki, Kai Ming Ting, and Akihiro Inokuchi, editors. Advances in Knowledge Discovery and Data Mining, Proceedings of the 12th Pacific-Asia Conference, PAKDD 2008, Osaka, Japan, May 20-23, volume 5012 of Lecture Notes in Computer Science. Springer, 2008. 